\title{
Directed migration of mesenchymal cells: where signaling and the cytoskeleton meet
}

\author{
James E. Bear ${ }^{1,3,{ }^{*}}$ and Jason M. Haugh ${ }^{2,{ }^{*}}$ \\ 1 UNC-Chapel Hill, UNC Lineberger Cancer Center and the Department of Cell Biology and \\ Physiology \\ ${ }^{2}$ North Carolina State University, Department of Chemical and Biomolecular Engineering \\ ${ }^{3}$ Howard Hughes Medical Institute, UNC-Chapel Hill
}

\begin{abstract}
Cell migration directed by spatial cues, or taxis, is a primary mechanism for orchestrating concerted and collective cell movements during development, wound repair, and immune responses. Compared with the classic example of amoeboid chemotaxis, in which fast-moving cells such as neutrophils are directed by gradients of soluble factors, directed migration of slowmoving mesenchymal cells such as fibroblasts is poorly understood. Mesenchymal cells possess a distinctive organization of the actin cytoskeleton and associated adhesion complexes as its primary mechanical system, generating the asymmetric forces required for locomotion without strong polarization. The emerging hypothesis is that the molecular underpinnings of mesenchymal taxis involve distinct signaling pathways and diverse requirements for regulation.
\end{abstract}

\section{Introduction}

Chemotaxis, or cell migration directed by an external chemical gradient, is a primary means of intercellular communication. For example, two very different examples of chemotaxis are encountered during the inflammatory and proliferative phases of cutaneous wound healing [1]. During the inflammatory phase, neutrophils and macrophages are recruited from the circulation by gradients of soluble and immobilized chemokines, and once in the wound, these cells move chemotactically to ingest debris and bacteria. This is a rapid process, established within hours. By comparison, the proliferative phase spans days to weeks and is characterized by the proliferation and relatively slow chemotactic migration of fibroblasts, which are recruited from the collagen-rich dermis into the fibrinogen- and fibronectin-rich provisional matrix of the clotted wound. The primary chemotactic signal for the invading fibroblasts is platelet-derived growth factor (PDGF), released by platelets and macrophages [2]. The role of PDGF as a chemoattractant generally translates to other mesenchymal

(C) 2014 Elsevier Ltd. All rights reserved.

*Address correspondence to JEB (jbear@email.unc.edu) or JMH (jason_haugh@ncsu.edu).

Publisher's Disclaimer: This is a PDF file of an unedited manuscript that has been accepted for publication. As a service to our customers we are providing this early version of the manuscript. The manuscript will undergo copyediting, typesetting, and review of the resulting proof before it is published in its final citable form. Please note that during the production process errors may be discovered which could affect the content, and all legal disclaimers that apply to the journal pertain. 
tissues (stroma), as seen in embryogenesis [3] and cardiovascular function [4,5]. PDGF signaling also plays a prominent role in tumorigenesis [6]. It is established that chemotactic signals influence cancer cell invasiveness, and thus metastasis, and growth factor signaling has been implicated in aggressiveness of mesenchymal tumors [7-10] and in reciprocal communication between carcinomas and nearby stromal cells [11,12]. In carcinomas, PDGF receptor signaling emerges in cancer stem cells following the epithelial-to-mesenchymal transition, a program associated with invasiveness [13•]. From these indications it is apparent that directed migration of mesenchymal cells is fundamentally important in both normal tissue homeostasis and in progression of disease.

Here, we examine evidence that characterizes mesenchymal chemotaxis, and other forms of directed migration exhibited by mesenchymal cells, as distinct from directed migration of leukocytes and other amoeboid cells. Whereas a common theme in cell locomotion is the generation of force applied in an asymmetric fashion, a mesenchymal cell exhibits unique architectures and dynamics of the actin cytoskeleton (and associated adhesion complexes) as its primary mechanical system. Accordingly, recent studies on mesenchymal cells suggest that signal transduction linking PDGF gradients and other spatial cues to local control of the actin cytoskeleton involves distinct molecular pathways and/or diverse requirements for regulation.

\section{Mesenchymal versus amoeboid migration}

Despite its pervasiveness in tissue development, homeostasis, and cancer, mesenchymal chemotaxis is poorly understood. Indeed, the bulk of the chemotaxis literature has focused on amoeboid cells such as neutrophils and the amoeba, Dictyostelium discoideum $[14,15]$. Amoeboid and mesenchymal motility modes lie at opposite extremes of cell migration phenotypes [16] and reflect the coordinated functions of the respective cell types (Fig. 1). The amoeboid migration phenotype is characterized by rapid locomotion (cell speed $\sim 10$ $\mu \mathrm{m} / \mathrm{min}$ ), a property attributed to the strong polarization that allows these cells to efficiently protrude via pseudopods and blebs and squeeze through pores in the connective tissue, largely unfettered by interactions with extracellular matrix (ECM) [17]. Amoeboid motility reflects the roles of neutrophils and lymphocytes as 'professional migrators' that must rapidly respond to crawl out of the circulation and then across great distances in secondary tissues to mediate innate and adaptive immunity, respectively [18]. In contrast, mesenchymal cells move slowly (cell speed $<1 \mu \mathrm{m} / \mathrm{min}$ ) and are weakly polarized, typically exhibiting multiple, competing protrusions (lamellipodia and filopodia) [19]. Another characteristic feature that limits the efficiency of mesenchymal motility is strong, integrinmediated adhesion to ECM. This 'friction' is tuned by the cells' ability to degrade matrix, through expression of matrix metalloproteinases, and to disassemble otherwise stable focal adhesions [20]. This reflects the intimate relationship between mesenchymal cells and matrix in general, exemplified by the role of fibroblasts in secretion and mechanochemical remodeling of ECM during wound repair.

Chemotactic gradient sensing is generally mediated by chemoattractant receptors of different types in amoeboid and mesenchymal cells. In neutrophils and lymphocytes, gradients of chemoattractants (e.g., chemokines such as IL-8, LTB4, CXCL12, and 
CXCL13, and N-formyl peptides shed by bacteria) are sensed by cognate receptors of the $\mathrm{G}$ protein-coupled receptor (GPCR) class. In fibroblasts, chemoattractants (certain growth factors, PDGF most notably but also fibroblast growth factors and epidermal growth factor (EGF)) are sensed by receptors of the receptor tyrosine kinase (RTK) class. Although it is true that the two classes of receptors largely access many of the same signaling pathways, i.e., those mediated by small GTPases and lipid second messengers, there are substantial differences in the dynamics of the two receptor types. A hallmark of GPCR regulation is desensitization, whereby agonist exposure results in rapid attenuation of the response. The theory of GPCR desensitization and adaptation as it relates to chemotaxis of amoeboid cells is well established. Adaptation is considered important for amoeboid cells' ability to sense the relative steepness of a chemoattractant gradient, allowing cells to respond over a broad range of absolute concentration [21,22]. Moreover, amoeboid cells are able to prioritize multiple chemoattractant cues and respond to them in sequence [23]. In a recent demonstration of this concept, primary neutrophils responding to gradients of IL-8 and LTB4 oriented in opposite directions oscillate/vacillate between the two, suggestive of switching between distinct sensing states [24•].

These receptor-level complexities do not have a known analog in RTK signaling. RTKs are subject to downregulation through the endosomal and ubiquitin-proteasome degradation pathways. As a consequence, in fibroblasts responding to a high dose of PDGF, tyrosine phosphorylation of its cognate receptors is transient on a time scale of $\sim 1$ hour [25]. Yet it is well known that prolonged exposure to growth factors is required to render cells competent for cell cycle progression, suggesting that receptor downregulation is simply a mass-action effect by which receptor expression is dampened when the growth factor concentration is far above physiological. Accordingly, it has been shown that multiple signal transduction pathways are prominently activated at sub-nanomolar concentrations of PDGF [25,26], which are far below receptor saturation and thus predicted to yield minimal receptor downregulation. Another established consequence of RTK endocytosis is growth factor (i.e., ligand) clearance. Mathematical modeling suggests that receptor-mediated clearance of PDGF might be important for maintaining a sharp gradient of the chemoattractant as fibroblasts collectively invade a wound [27]. Experimental evidence in a different chemotactic context, midbrain development in zebrafish $[28 \bullet, 29 \bullet]$, supports this concept.

Mesenchymal and amoeboid cells show certain similarities but also striking differences in their cytoskeletal organization that directly relate to their different modes of migration. Both cell types use the Arp2/3 complex to build dendritic arrays of actin filaments at their protruding edge(s). The Arp2/3 complex nucleates new filaments as branch points from existing ones and is a primary means of actin polymerization in lamellipodia [30,31]. Interspersed with the branched actin filaments are bundled, unbranched actin arrays that often protrude as finger-like filopodia. Alternate actin assembly pathways, such as those orchestrated by formins and Ena/VASP proteins, produce these structures [31,32]. These non-Arp2/3-based actin assembly pathways have been functionally linked to chemotaxis in neutrophils and tumor cell lines [33,34,35•]. Away from the leading edge, actin structures such as acto-myosin contractile arrays are quite different between mesenchymal and amoeboid cells. In amoeboid cells, Myosin II is primarily confined to the rear of the cells in a structure known as the uropod and is thought to provide a squeezing force that is both 
functionally coupled to actin polymerization-based protrusion at the front of the cell [36] and critical for bleb-based protrusions [16]. In mesenchymal cells, Myosin II is associated with bundled actin stress fibers; whereas the Myosin IIB isoform is located mainly in retracting regions of the cell, Myosin IIA is found throughout the cell [37]. Contractile actomyosin stress fibers are required for the strong substrate adhesion observed in mesenchymal cells and play a central role in regulation of membrane protrusion and overall cell migration [37-39]. Yet, the contribution of acto-myosin regulation to chemotaxis is poorly understood.

\section{Role of the PI3K/Rac/WAVE/Arp2/3 circuit: directional sensing or efficient movement?}

One mechanistic insight that initially seemed to unify the gradient sensing mechanisms of eukaryotic chemotaxis is receptor-mediated recruitment and activation of type I phosphoinositide 3-kinases (PI3Ks). These enzymes produce the lipid second messenger $\mathrm{PIP}_{3}$ at the plasma membrane, and $\mathrm{PIP}_{3}$ is readily dephosphorylated to form other phosphoinositides. Although GPCRs and RTKs mediate activation of differentially regulated isoforms of the PI3K enzyme, a common feature of the pathway in chemotaxing cells is formation of a spatially asymmetric pattern, with higher densities of 3 ' phosphoinositides biased in the direction of the chemoattractant gradient $[22,40,41]$. Another common signaling intermediate that generally exhibits bias in the direction of cell locomotion is the active, GTP-bound form of Rac [42,43]. PIP 3 and Rac-GTP each target multiple effectors and thus can promote cell migration in both subtle and direct ways; arguably the most direct is their synergistic recruitment and activation of the WAVE regulatory complex, which in turn activates the Arp2/3 complex [44]. Among the other targets of $\mathrm{PIP}_{3}$ are certain guanine nucleotide exchange factors (GEFs) that mediate increases in Rac-GTP, and in turn Rac-GTP can promote PI3K signaling [43,45,46], in theory suggesting a complex signaling circuit with coherent feedforward and/or positive feedback loops that are thought to endow amplified sensitivity and robust polarization of signaling during chemotaxis $[36,47,48,49 \bullet]$. Based on these insights it has been assumed that activation of this signaling circuit leading to focal enhancement of Arp2/3-mediated F-actin polymerization is a common basis for gradient sensing, i.e., the chemotactic 'compass', perhaps with subtle variations across cell types and chemoattractant gradient conditions [40,50] (Fig. 2A).

In the context of mesenchymal chemotaxis in particular, various lines of evidence challenge this model. One is the use of direct observation chemotaxis chambers and cell tracking, which, together with advances in molecular interventions such as RNA interference, trump previous methods such as Boyden chamber assays. Recent studies indicate that neither PI3K [51] nor Rac [52] is absolutely required for PDGF chemotaxis. Modulation of these signaling intermediates affects cell morphology and migration speed, and even subtle changes in Rac signaling alters persistence of randomly migrating fibroblasts $[53,54 \cdot]$. But the ability to sense and respond chemotactically to a PDGF gradient is not grossly affected. Even more compelling is the observation that fibroblasts depleted of Arp2/3 complex, which completely lack dendritic F-actin and lamellipodia, show reduced cell speed but no change in fidelity of PDGF chemotaxis [55••]. Although another study implicated the Arp2/3 complex as essential for EGF chemotaxis [56•], the discrepancy with the abovementioned PDGF study can be attributed to differences in chemotactic chamber design and a non- 
autonomous effect of factors secreted by Arp2/3-deficient cells [57•]. In weakly polarized mesenchymal cells, the signaling circuit leading to Arp2/3 activation is apparently required for efficient locomotion but not for gradient sensing (Fig. 2B).

Studies employing live-cell imaging of fluorescent protein biosensors support this alternative view. Localization of active Rac consistently accumulates after, not before, the onset of leading-edge protrusion [58,59]. Local Rac signaling is clearly sufficient to drive membrane protrusion and directed migration $[46,60]$, so how are these observations reconciled? We recently showed that the role of PI3K signaling in fibroblasts is not to initiate protrusion but rather to stabilize nascent lamellipodia; the propagation of this process manifests as branching of lamellipodia and large-scale reorientation of migration directionality, which allows fibroblasts to efficiently align their locomotion towards a PDGF gradient [61•]. The implication is that PI3K and Rac signaling are important amplifiers that drive the engine of cell motility, but in mesenchymal cells they take their cue from a different process. As the search continues for signaling pathways that are required for mesenchymal chemotaxis, what is clear is that PI3K and Rac signaling are not simply redundant, i.e., replaceable ways to achieve gradient sensing by converging on the Arp $2 / 3$ complex. A wholly different means of asymmetric force generation must be at play. For example, PDGF receptor signaling might mobilize actin nucleators other than Arp2/3 complex or regulate myosin contractility (Fig. 2B). A clue supporting the latter possibility is the observation that PDGF stimulation of fibroblasts reduces RhoA activity at the cell front [62].

\section{Directed migration towards a diverse set of spatial cues}

While recent studies have seemingly yielded more questions than answers about the mechanisms of mesenchymal chemotaxis, even less is understood about other forms of directed migration that mesenchymal cells exhibit [63] (Fig. 3A). Given the aforementioned importance of ECM in their physiology, it is not surprising that mesenchymal cells engage in haptotaxis, or migration biased by a gradient of immobilized ligands. Adhesive ligands in ECM are recognized by various integrins that cluster to form nascent adhesion complexes under lamellipodia, some of which grow to form mature focal adhesions that are mechanically coupled to large, contractile actin stress fibers. In addition to these differences in mechanical linkages between the ECM and F-actin, nascent and mature adhesions have different signal transduction properties, with nascent adhesions mediating Rac signaling and thus Arp2/3-based lamellipodial protrusion [64-66]. Interestingly, whereas the Arp2/3 complex is dispensable for PDGF chemotaxis in fibroblasts, it is absolutely required for haptotaxis towards a variety of ECM cues [55••]. In haptotaxis, the cell must actively send out protrusions to encounter anchored ligands, while in chemotaxis, ligand molecules are encountered passively by diffusion. This difference might explain the requirement for proper protrusive structures and dynamics for successful haptotaxis. A hybrid form of chemotaxis and haptotaxis also exists, in which growth factors and chemokines that can function as soluble cues also bind to ECM and direct migration as immobilized ligands [67•,68••]. Whether this mode of directed migration is more similar to chemotaxis or haptotaxis in terms of molecular requirements remains to be determined. 
Cell migration is also directed by mechanical and electrical cues. Certain cell types have the ability to sense and respond to a gradient of mechanical stiffness, a process known as durotaxis or mechanotaxis [69-71]. Mesenchymal cells are unique because the variable stiffness of their microenvironment is largely a property of the ECM (thus, durotaxis and haptotaxis are related), and because of the magnitude of the traction forces that they exert [72]. Recent work points toward the mechanical sensitivity of focal adhesions in durotactic responses [73••]. Cyclic fluctuations in force produced at focal adhesions allow cells to tug on flexible substrates and gauge relative stiffness. The regulation of several focal adhesion components, including FAK, vinculin, and paxillin, are critical for this durotactic sensing. A possible mechanotransduction mechanism is the activation of Rho, which is mediated by adhesions under tension, leading to activation of myosin contractility [74•]. Indeed, in mesenchymal stem cells migrating from soft to stiff matrix, there is a dramatic change in Myosin IIA/B organization [75•]. In future work, it will be exciting to see the process of durotaxis examined in more physiological settings and in other cell types, particularly those lacking classical focal adhesions. In addition, it will be interesting to study the interplay between haptotaxis and durotaxis when both ECM ligand density and mechanical compliance are being sensed. Cells also respond to electrical gradients (electrotaxis or galvanotaxis) in situations such as wound healing. Sensing this type of cue does not require ionic flux across the membrane, but it does seem to require electrophoretic displacement of membrane components and some intracellular signaling pathways such as PI3K [76,77•].

It is important to note that cells engaged in directed migration in vivo likely encounter multiple types of cues that they must simultaneously evaluate and prioritize to achieve an appropriate physiological response. For mesenchymal cells, two situations where directed migration plays a significant role are cutaneous wound healing and tumor cell invasion following epithelial-to-mesenchymal transition (Fig. 3B,C). During wound healing, dermal fibroblasts migrate into the clotted wound in order to reorganize and resynthesize the matrix. PDGF emanating from platelets and macrophages in the provisional matrix is a critical directional cue for these cells, but it seems likely that haptotactic, durotactic, and galvanotactic cues also play a role in fibroblast recruitment. How these cues might act in concert remains an open question, but some evidence indicates that direct crosstalk between integrin and PDGF signaling regulates mesenchymal stem cell migration [78]. Similarly, during tumor progression, a subpopulation of tumor cells adopt an invasive, mesenchymal phenotype and migrate away from the primary tumor [79]. These cells migrate towards blood and lymphatic vessels as part of the metastatic cascade of tumor dissemination. The directional migration cues in these situations are incompletely understood, but in the case of mammary adenocarcinoma these cells are responding to EGF cues released by tissue macrophages [80] as well as mechanical stiffness of the surrounding matrix [81,82]. Much remains to be learned about directed migration during pathophysiological situations such as metastatic cancer.

\section{Unifying principles of directed migration}

A guiding principle that unifies all forms of directed migration is asymmetric force generation aligned with the extracellular cue. In some cells, this is likely achieved by localized actin polymerization at the leading edge. However, other sources of asymmetric 
force, such as differential adhesion, myosin motor activity, or osmotic pressure [83•] could serve this purpose. A second principle is the ability to spatially or/and temporally sense variations in the external environment, and to link that sensing via signal transduction to actuate a mechanical response. Hence, understanding directed cell migration will require greater focus on the interface between signaling and cytoskeletal networks.

\section{Acknowledgments}

We gratefully acknowledge support from HHMI, NIH grant to JEB (GM083035), and NSF grant to JMH (1133476), and we apologize to those authors whose excellent work could not be cited due to space limitations.

\section{References}

- Special interest

•• Outstanding interest

1. Singer AJ, Clark RAF. Mechanisms of disease: cutaneous wound healing. N Engl J Med. 1999; 341:738-746. [PubMed: 10471461]

2. Deuel TF, Kawahara RS, Mustoe TA, Pierce GF. Growth factors and wound healing: plateletderived growth factor as a model cytokine. Annu Rev Med. 1991; 42:567-584. [PubMed: 2035994]

3. Ataliotis P, Mercola M. Distribution and functions of platelet-derived growth factors and their receptors during embryogenesis. Intl Rev Cytol. 1997; 172:95-127.

4. Edelberg JM, Cai D, Xaymardan M. Translation of PDGF cardioprotective pathways. Cardiovasc Toxicol. 2003; 3:27-35. [PubMed: 12668888]

5. Leask A. Potential therapeutic targets for cardiac fibrosis: $\mathrm{TGFb}$, angiotensin, endothelin, $\mathrm{CCN} 2$, and PDGF, partners in fibroblast activation. Circ Res. 2010; 106:1675-1680. [PubMed: 20538689]

6. Ostman A, Heldin CH. PDGF receptors as targets in tumor treatment. Adv Cancer Res. 2007; 97:247-274. [PubMed: 17419949]

7. Cao RH, Bjorndahl MA, Religa P, Clasper S, Garvin S, Galter D, Meister B, Ikomi F, Tritsaris K, Dissing S, et al. PDGF-BB induces intratumoral lymphangiogenesis and promotes lymphatic metastasis. Cancer Cell. 2004; 6:333-345. [PubMed: 15488757]

8. Ingram JL, Bonner JC. EGF and PDGF receptor tyrosine kinases as therapeutic targets for chronic lung diseases. Curr Mol Med. 2006; 6:409-421. [PubMed: 16900664]

9. Shih AH, Holland EC. Platelet-derived growth factor (PDGF) and glial tumorigenesis. Cancer Lett. 2006; 232:139-147. [PubMed: 16139423]

10. Andrae J, Gallini R, Betsholtz C. Role of platelet-derived growth factors in physiology and medicine. Genes Dev. 2008; 22:1276-1312. [PubMed: 18483217]

11. Micke P, Ostman A. Exploring the tumour environment: cancer-associated fibroblasts as targets in cancer therapy. Expert Opin Ther Targets. 2005; 9:1217-1233. [PubMed: 16300472]

12. Bremnes RM, Dønnem T, Al-Saad S, Al-Shibli K, Andersen S, Sirera R, Camps C, Marinez I, Busund LT. The role of tumor stroma in cancer progression and prognosis: Emphasis on carcinoma-associated fibroblasts and non-small cell lung cancer. J Thorac Oncol. 2011; 6:209217. [PubMed: 21107292]

13. Tam WL, Lu H, Buikhuisen J, Soh BS, Lim E, Reinhardt F, Wu ZJ, Krall JA, Bierie B, Guo W, et al. Protein kinase $\mathrm{C}$ alpha is a central signaling node and therapeutic target for breast cancer stem cells. Cancer Cell. 2013; 24:347-364. [PubMed: 24029232] [This paper describes a fascinating switch in signaling in cancer stem cells towards a dependence on PDGF/PKCa and away from EGF-based signaling, which occurs in the epithelial-to-mesenchymal transition. The study provides new evidence highlighting the importance of PDGF signaling in cancer and implicates signaling pathways that might be targeted to treat aggressive breast cancers in particular.] 
14. Swaney KF, Huang CH, Devreotes PN. Eukaryotic chemotaxis: a network of signaling pathways controls motility, directional sensing, and polarity. Annu Rev Biophys. 2010; 39:265-289. [PubMed: 20192768]

15. Insall RH. Understanding eukaryotic chemotaxis: a pseudopod-centred view. Nat Rev Mol Cell Biol. 2010; 11:453-458. [PubMed: 20445546]

16. Friedl P, Wolf K. Plasticity of cell migration: a multiscale tuning model. J Cell Biol. 2010; 188:11-19. [PubMed: 19951899]

17. Lämmermann T, Bader BL, Monkley SJ, Worbs T, Wedlich-Soldner R, Hirsch K, Keller M, Forster R, Critchley DR, Fässler R, et al. Rapid leukocyte migration by integrin-independent flowing and squeezing. Nature. 2008; 453:51-55. [PubMed: 18451854]

18. Campbell DJ, Kim CH, Butcher EC. Chemokines in the systemic organization of immunity. Immunol Rev. 2003; 195:58-71. [PubMed: 12969310]

19. Petrie RJ, Doyle AD, Yamada KM. Random versus directionally persistent cell migration. Nat Rev Mol Cell Biol. 2009; 10:538-549. [PubMed: 19603038]

20. Even-Ram S, Yamada KM. Cell migration in 3D matrix. Curr Opin Cell Biol. 2005; 17:524-532. [PubMed: 16112853]

21. Fisher PR, Merkl R, Gerisch G. Quantitative analysis of cell motility and chemotaxis in Dictyostelium discoideum by using an image processing system and a novel chemotaxis chamber providing stationary chemical gradients. J Cell Biol. 1989; 108:973-984. [PubMed: 2537839]

22. Parent CA, Devreotes PN. A cell's sense of direction. Science. 1999; 284:765-770. [PubMed: 10221901]

23. Foxman EF, Kunkel EJ, Butcher EC. Integrating conflicting chemotactic signals: the role of memory in leukocyte navigation. J Cell Biol. 1999; 147:577-587. [PubMed: 10545501]

24•. Byrne MB, Kimura Y, Kapoor A, He Y, Mattam KS, Hasan KM, Olson LN, Wang F, Kenis PJ, Rao CV. Oscillatory behavior of neutrophils under opposing chemoattractant gradients supports a winner-take-all mechanism. PLoS ONE. 2014; 9:e85726. [PubMed: 24465668] [In this study, a microfluidic device is used to establish opposing gradients of two chemoattractants, IL-8 and LTB4. In this system, primary neutrophils vacillated between directions rather than stalling at a point where sensing of the two gradients is equivalent. The authors combine these observations with computational modeling to propose a mechanism that switches between stable states, in which sensing of one or the other gradient dominates. This implies a level of complexity that is encoded at the level of the receptors involved, which are of the GPCR class.]

25. Park CS, Schneider IC, Haugh JM. Kinetic analysis of platelet-derived growth factor receptor/ phosphoinositide 3-kinase/Akt signaling in fibroblasts. J Biol Chem. 2003; 278:37064-37072. [PubMed: 12871957]

26. Wang C-C, Cirit M, Haugh JM. PI3K-dependent crosstalk interactions converge with Ras as quantifiable inputs integrated by Erk. Mol Syst Biol. 2009; 5:246. [PubMed: 19225459]

27. Haugh JM. Deterministic model of dermal wound invasion incorporating receptor-mediated signal transduction and spatial gradient sensing. Biophys J. 2006; 90:2297-2308. [PubMed: 16415056]

28•. Lewellis SW, Nagelberg D, Subedi A, Staton A, LeBlanc M, Giraldez A, Knaut H. Precise SDF1mediated cell guidance is achieved through ligand clearance and microRNA-mediated decay. $\mathrm{J}$ Cell Biol. 2013200:337-355. [PubMed: 23382464] [See annotation of Dona et al. (2013) [29•].]

29•. Dona E, Barry JD, Velentin G, Quirin C, Khmelinskii A, Kunze A, Durdu S, Newton LR, Fernandez-Milan A, Huber W, et al. Directional tissue migration through a self-generated chemokine gradient. Nature. 2013; 503:285-289. [PubMed: 24067609] [These two studies offer evidence supporting the conceptual idea that receptor-mediated clearance of chemoattractant sharpens the external gradient and thus promotes chemotaxis over long distances. Haugh analyzed similar dynamics in a mathematical model of PDGF chemotaxis during wound invasion (ref. 27). The SDF1 chemotaxis and clearance in zebrafish are mediated by GPCRs, suggesting that ligand clearance may be a common feature of collective cell invasion processes.]

30. Rotty JD, Wu C, Bear JE. New insights into the regulation and cellular functions of the ARP2/3 complex. Nat Rev Mol Cell Biol. 2013; 14:7-12. [PubMed: 23212475]

31. Campellone KG, Welch MD. A nucleator arms race: cellular control of actin assembly. Nat Rev Mol Cell Biol. 2010; 11:237-251. [PubMed: 20237478] 
32. Bear JE, Gertler FB. Ena/VASP: towards resolving a pointed controversy at the barbed end. J Cell Sci. 2009; 122:1947-1953. [PubMed: 19494122]

33. Neel NF, Barzik M, Raman D, Sobolik-Delmaire T, Sai J, Ham AJ, Mernaugh RL, Gertler FB, Richmond A. VASP is a CXCR2-interacting protein that regulates CXCR2-mediated polarization and chemotaxis. J Cell Sci. 2009; 122:1882-1894. [PubMed: 19435808]

34. Shi Y, Zhang J, Mullin M, Dong B, Alberts AS, Siminovitch KA. The mDial formin is required for neutrophil polarization, migration, and activation of the LARG/RhoA/ROCK signaling axis during chemotaxis. J Immunol. 2009; 182:3837-3845. [PubMed: 19265163]

35. Daou P, Hasan S, Breitsprecher D, Baudelet E, Camoin L, Audebert S, Goode BL, Badache A. Essential and nonredundant roles for Diaphanous formins in cortical microtubule capture and directed cell migration. Mol Biol Cell. 2014; 25:658-668. [PubMed: 24403606] [Daou et al. show that depletion of any one of three formins, mDia1, mDia2, or mDia3, impairs chemotaxis of a cancer cell line. Remarkably, it does so without noticeably altering F-actin structures; instead, distinct roles in microtubule dynamics are implicated.]

36. Xu J, Wang F, Van Keymeulen A, Herzmark P, Straight A, Kelly K, Takuwa Y, Sugimoto N, Mitchison T, Bourne HR. Divergent signals and cytoskeletal assemblies regulate self-organizing polarity in neutrophils. Cell. 2003; 114:201-214. [PubMed: 12887922]

37. Vicente-Manzanares M, Zareno J, Whitmore L, Choi CK, Horwitz AF. Regulation of protrusion, adhesion dynamics, and polarity by myosins IIA and IIB in migrating cells. J Cell Biol. 2007; 176:573-580. [PubMed: 17312025]

38. Even-Ram S, Doyle AD, Conti MA, Matsumoto K, Adelstein RS, Yamada KM. Myosin IIA regulates cell motility and actomyosin-microtubule crosstalk. Nat Cell Biol. 2007; 9:299-309. [PubMed: 17310241]

39. Vicente-Manzanares M, Newell-Litwa K, Bachir AI, Whitmore LA, Horwitz AR. Myosin IIA/IIB restrict adhesive and protrusive signaling to generate front-back polarity in migrating cells. J Cell Biol. 2011; 193:381-396. [PubMed: 21482721]

40. Weiner OD. Regulation of cell polarity during eukaryotic chemotaxis: the chemotactic compass. Curr Opin Cell Biol. 2002; 14:196-202. [PubMed: 11891119]

41. Schneider IC, Haugh JM. Quantitative elucidation of a distinct spatial gradient-sensing mechanism in fibroblasts. J Cell Biol. 2005; 171:883-892. [PubMed: 16314431]

42. Kraynov VS, Chamberlain C, Bokoch GM, Schwartz MA, Slabaugh S, Hahn KM. Localized Rac activation dynamics visualized in living cells. Science. 2000; 290:333-337. [PubMed: 11030651]

43. Srinivasan S, Wang F, Glavas S, Ott A, Hofmann F, Aktories K, Kalman D, Bourne HR. Rac and $\mathrm{Cdc} 42$ play distinct roles in regulating $\mathrm{PI}(3,4,5) \mathrm{P} 3$ and polarity during neutrophil chemotaxis. $\mathbf{J}$ Cell Biol. 2003; 160:375-385. [PubMed: 12551955]

44. Lebensohn AM, Kirschner MW. Activation of the WAVE complex by coincident signals controls actin assembly. Mol Cell. 2009; 36:512-524. [PubMed: 19917258]

45. Weiner OD, Neilsen PO, Prestwich GD, Kirschner MW, Cantley LC, Bourne HR. A PtdInsP3- and Rho GTPase-mediated positive feedback loop regulates neutrophil polarity. Nat Cell Biol. 2002; 4:509-512. [PubMed: 12080346]

46. Yoo SK, Deng Q, Cavnar PJ, Wu YI, Hahn KM, Huttenlocher A. Differential regulation of protrusion and polarity by PI3K during neutrophil motility in live zebrafish. Dev Cell. 2010; 18:226-236. [PubMed: 20159593]

47. Wong K, Pertz O, Hahn K, Bourne HR. Neutrophil polarization: spatiotemporal dynamics of RhoA activity support a self-organizing mechanism. Proc Natl Acad Sci USA. 2006; 103:3639-3644. [PubMed: 16537448]

48 . Jilkine A, Edelstein-Keshet L. A comparison of mathematical models for polarization of single eukaryotic cells in response to guided cues. PLoS Comput Biol. 2011; 7:e1001121. [PubMed: 21552548] [Live-cell microscopy and mathematical modeling are combined in this quantitative study of D. discoideum migration stimulated by cAMP. The authors show that the classic actin polymerization mechanism involving the Arp2/3 complex exhibits rapid but weak oscillations until excited by upstream signaling, e.g., through PI3K and Rac. The paper offers a hypothetical mechanism wherein sustained protrusion occurs only when a signaling threshold is exceeded and 
thus provides a foundation for understanding the coupling between signaling and cytoskeletal dynamics.]

49. Huang CH, Tang M, Shi C, Iglesias PA, Devreotes PN. An excitable signal integrator couples to an idling cytoskeletal oscillator to drive cell migration. Nat Cell Biol. 2013; 15:1307-1316. [PubMed: 24142103]

50. Arrieumerlou C, Meyer T. A local coupling model and compass parameter for eukaryotic chemotaxis. Dev Cell. 2005; 8:215-227. [PubMed: 15691763]

51. Melvin AT, Welf ES, Wang Y, Irvine DJ, Haugh JM. In chemotaxing fibroblasts, both highfidelity and weakly biased cell movements track the localization of PI3K signaling. Biophys J. 2011; 100:1893-1901. [PubMed: 21504725]

52. Monypenny J, Zicha D, Higashida C, Oceguera-Yanez F, Narumiya S, Watanabe N. Cdc42 and Rac family GTPases regulate mode and speed but not direction of primary fibroblast migration during platelet-derived growth factor-dependent chemotaxis. Mol Cell Biol. 2009; 29:2730-2747. [PubMed: 19273601]

53. Pankov R, Endo Y, Even-Ram S, Araki M, Clark K, Cukierman E, Matsumoto K, Yamada KM. A Rac switch regulates random versus directionally persistent cell migration. J Cell Biol. 2005; 170:793-802. [PubMed: 16129786]

54- Dang I, Gorelik R, Sousa-Blin C, Derivery E, Guerin C, Linkner J, Nemethova M, Dumortier JG, Giger FA, Chipysheva TA, et al. Inhibitory signalling to the Arp2/3 complex steers cell migration. Nature. 2013; 503:281-284. [PubMed: 24132237] [This paper describes the discovery and establishes a putative role of Arpin, a protein that inhibits the Arp2/3 complex in response to Rac signaling. Consistent with previous indications in ref. 53 by Pankov et al., modulation of the Rac/WAVE/Arp2/3 pathway via Arpin affects migration persistence in a variety of cell types. The study implicates an incoherent feedforward loop in which different Rac effectors positively and negatively regulate Arp2/3-mediated protrusion.]

55••. Wu C, Asokan SB, Berginski ME, Haynes EM, Sharpless NE, Griffith JD, Gomez SM, Bear JE. Arp $2 / 3$ is critical for lamellipodia and response to extracellular matrix cues but is dispensable for chemotaxis. Cell. 2012; 148:973-987. [PubMed: 22385962] [This paper describes the effect of Arp2/3 complex depletion on fibroblast chemotaxis and haptotaxis. The surprising finding is that Arp2/3 depletion, which leaves no detectable lamellipodia, reduces cell migration speed but does not affect the fidelity of PDGF chemotaxis. This result forces us to evaluate alternative signaling and force generation mechanisms. At the same time, Arp2/3 depletion blocks ECM haptotaxis, indicating diverse requirements for different forms of directed migration.]

56•. Suraneni P, Rubinstein B, Unruh JR, Durnin M, Hanein D, Li R. The Arp2/3 complex is required for lamellipodia extension and directional fibroblast cell migration. J Cell Biol. 2012197:239251. [PubMed: 22492726] [See annotation of Wu et al. (2013) [57•].]

57•. Wu C, Haynes EM, Asokan SB, Simon JM, Sharpless NE, Baldwin AS, Davis IJ, Johnson GL, Bear JE. Loss of Arp2/3 induces an NF-kappaB-dependent, nonautonomous effect on chemotactic signaling. J Cell Biol. 2013; 203:907-916. [PubMed: 24344184] [These papers illustrate that the ability of fibroblasts to chemotax after loss of Arp $2 / 3$ is context-dependent. Suraneni et al. showed that cells lacking Arp2/3 complex could not chemotax towards EGF in a stagnant, non-perfused chamber. Wu et al. subsequently showed that loss of Arp2/3 induces secretion of factors that mask gradient sensing unless there is exchange of medium by convective flow.]

58. Tsukada Y, Aoki K, Nakamura T, Sakumura Y, Matsuda M, Ishii S. Quantification of local morphodynamics and local GTPase activity by edge evolution tracking. PLoS Comput Biol. 2008; 4

59. Machacek M, Hodgson L, Welch C, Elliott H, Pertz O, Nalbant P, Abell A, Johnson GL, Hahn KM, Danuser G. Coordination of Rho GTPase activities during cell protrusion. Nature. 2009; 461:99-103. [PubMed: 19693013]

60. Wu YI, Frey D, Lungu OI, Jaehrig A, Schlichting I, Kuhlman B, Hahn KM. A genetically encoded photoactivatable Rac controls the motility of living cells. Nature. 2009; 461:104-108. [PubMed: 19693014]

61 • Welf ES, Ahmed S, Johnson HE, Melvin AT, Haugh JM. Migrating fibroblasts reorient directionality by a metastable, PI3K-dependent mechanism. J Cell Biol. 2012; 197:105-114. 
[PubMed: 22472441] [This paper shows how fibroblasts reorient migration by branching of lamellipodia. In this process, PI3K signaling is required for the stabilization and propagation of the nascent lamellipodia formed after initiation of a branch. The study clarifies the role of PI3K signaling in fibroblast migration; although not absolutely required for PDGF chemotaxis, this pathway is needed for efficient reorientation and facilitates alignment of cells with an external PDGF gradient.]

62. Pertz O, Hodgson L, Klemke RL, Hahn KM. Spatiotemporal dynamics of RhoA activity in migrating cells. Nature. 2006; 440:1069-1072. [PubMed: 16547516]

63. Lara Rodriguez L, Schneider IC. Directed cell migration in multi-cue environments. Integr Biol (Camb). 2013; 5:1306-1323. [PubMed: 24089107]

64. Beningo KA, Dembo M, Kaverina I, Small JV, Wang YL. Nascent focal adhesions are responsible for the generation of strong propulsive forces in migrating fibroblasts. J Cell Biol. 2001; 153:881888. [PubMed: 11352946]

65. Cox EA, Sastry SK, Huttenlocher A. Integrin-mediated adhesion regulates cell polarity and membrane protrusion through the Rho family of GTPases. Mol Biol Cell. 2001; 12:265-277. [PubMed: 11179414]

66. Nayal A, Webb DJ, Brown CM, Schaefer EM, Vicente-Manzanares M, Horwitz AR. Paxillin phosphorylation at Ser273 localizes a GIT1-PIX-PAK complex and regulates adhesion and protrusion dynamics. J Cell Biol. 2006; 173:587-599. [PubMed: 16717130]

67•. Haessler U, Pisano M, Wu M, Swartz MA. Dendritic cell chemotaxis in 3D under defined chemokine gradients reveals differential response to ligands CCL21 and CCL19. Proc Natl Acad Sci U S A. 2011108:5614-5619. [PubMed: 21422278] [See annotation of Weber et al. (2013) [68••].]

68••. Weber M, Hauschild R, Schwarz J, Moussion C, de Vries I, Legler DF, Luther SA, Bollenbach T, Sixt M. Interstitial dendritic cell guidance by haptotactic chemokine gradients. Science. 2013; 339:328-332. [PubMed: 23329049] [These papers evaluate directed migration of dendritic cells by the CCR7 ligands, CCL21 and CCL19, in realistic microenvironments. Haessler et al. used a microfluidic device to establish chemokine gradients across a 3D matrix; they found that CCL21 was more potent than CCL19 and attributed this difference to the immobilization of CCL21 by matrix proteoglycans. Weber et al. later showed that dendritic cells respond to gradients of immobilized CCL21 presented in vivo.]

69. Lo CM, Wang HB, Dembo M, Wang YL. Cell movement is guided by the rigidity of the substrate. Biophys J. 2000; 79:144-152. [PubMed: 10866943]

70. Isenberg BC, DiMilla PA, Walker M, Kim S, Wong JY. Vascular smooth muscle cell durotaxis depends on substrate stiffness gradient strength. Biophys J. 2009; 97:1313-1322. [PubMed: 19720019]

71. Roca-Cusachs P, Sunyer R, Trepat X. Mechanical guidance of cell migration: lessons from chemotaxis. Current Opinion in Cell Biology. 2013; 25:543-549. [PubMed: 23726023]

72. Munevar S, Wang YL, Dembo M. Traction force microscopy of migrating normal and H-ras transformed 3T3 fibroblasts. Biophys J. 2001; 80:1744-1757. [PubMed: 11259288]

73••. Plotnikov SV, Pasapera AM, Sabass B, Waterman CM. Force fluctuations within focal adhesions mediate ECM-rigidity sensing to guide directed cell migration. Cell. 2012; 151:1513-1527. [PubMed: 23260139] [This paper examines the role of force fluctuations, detected at the level of individual focal adhesions, on durotactic sensing. It implicates key focal adhesion components, namely FAK, vinculin, and paxillin, in this process. Interestingly, the ability of the cell to tug on ECM-coated substrata was found to be required for durotaxis but not haptotaxis.]

74•. Guilluy C, Swaminathan V, Garcia-Mata R, O'Brien ET, Superfine R, Burridge K. The Rho GEFs LARG and GEF-H1 regulate the mechanical response to force on integrins. Nat Cell Biol. 201113:722-727. [PubMed: 21572419] [See the annotation for Raab et al. (2012) [75•].]

75•. Raab M, Swift J, Dingal P, Shah P, Shin JW, Discher DE. Crawling from soft to stiff matrix polarizes the cytoskeleton and phosphoregulates myosin-II heavy chain. J Cell Biol. 2012; 199:669-683. [PubMed: 23128239] [These papers implicate Rho signaling and organization of Myosin II isoforms in mechanosensing and durotaxis. Guilluy et al. revealed a mechanism wherein adhesions under tension activate Rho signaling through LARG and GEF-H1. Given that Rho/ROCK signaling is a prominent pathway for activation of Myosin II, and hence an increase 
in tension, these findings suggest a basis for a positive feedback loop that reins in membrane protrusion. In a different context, Raab et al. showed that Myosin II transitions from a diffuse to an organized subcellular distribution as cells migrate from soft to stiff matrix.]

76. Zhao M, Song B, Pu J, Wada T, Reid B, Tai G, Wang F, Guo A, Walczysko P, Gu Y, et al. Electrical signals control wound healing through phosphatidylinositol-3-OH kinase-gamma and PTEN. Nature. 2006; 442:457-460. [PubMed: 16871217]

77•. Allen GM, Mogilner A, Theriot JA. Electrophoresis of cellular membrane components creates the directional cue guiding keratocyte galvanotaxis. Curr Biol. 2013; 23:560-568. [PubMed: 23541731] [This paper demonstrates galvanotaxis (electrotaxis) of fish keratocytes and implicates electrophoresis of membrane proteins and PI3K signaling in the underlying mechanism.]

78. Veevers-Lowe J, Ball SG, Shuttleworth A, Kielty CM. Mesenchymal stem cell migration is regulated by fibronectin through alpha5beta1-integrin-mediated activation of PDGFR-beta and potentiation of growth factor signals. J Cell Sci. 2011; 124:1288-1300. [PubMed: 21429937]

79. Guarino M, Rubino B, Ballabio G. The role of epithelial-mesenchymal transition in cancer pathology. Pathology. 2007; 39:305-318. [PubMed: 17558857]

80. Roussos ET, Condeelis JS, Patsialou A. Chemotaxis in cancer. Nat Rev Cancer. 2011; 11:573-587. [PubMed: 21779009]

81. Schedin P, Keely PJ. Mammary gland ECM remodeling, stiffness, and mechanosignaling in normal development and tumor progression. Cold Spring Harb Perspect Biol. 2011; 3:a003228. [PubMed: 20980442]

82. Lu P, Weaver VM, Werb Z. The extracellular matrix: a dynamic niche in cancer progression. J Cell Biol. 2012; 196:395-406. [PubMed: 22351925]

83•. Stroka KM, Jiang H, Chen SH, Tong Z, Wirtz D, Sun SX, Konstantopoulos K. Water permeation drives tumor cell migration in confined microenvironments. Cell. 2014; 157:611-623. [PubMed: 24726433] [This study combines imaging experiments and mathematical modeling to explain the mechanism of tumor cell migration in confined environments. It is shown that cell locomotion can be driven by an osmotic pressure gradient, achieved by polarizing the distribution of ion pumps and aquaporins, even when actin polymerization and myosin contractility are inhibited.] 


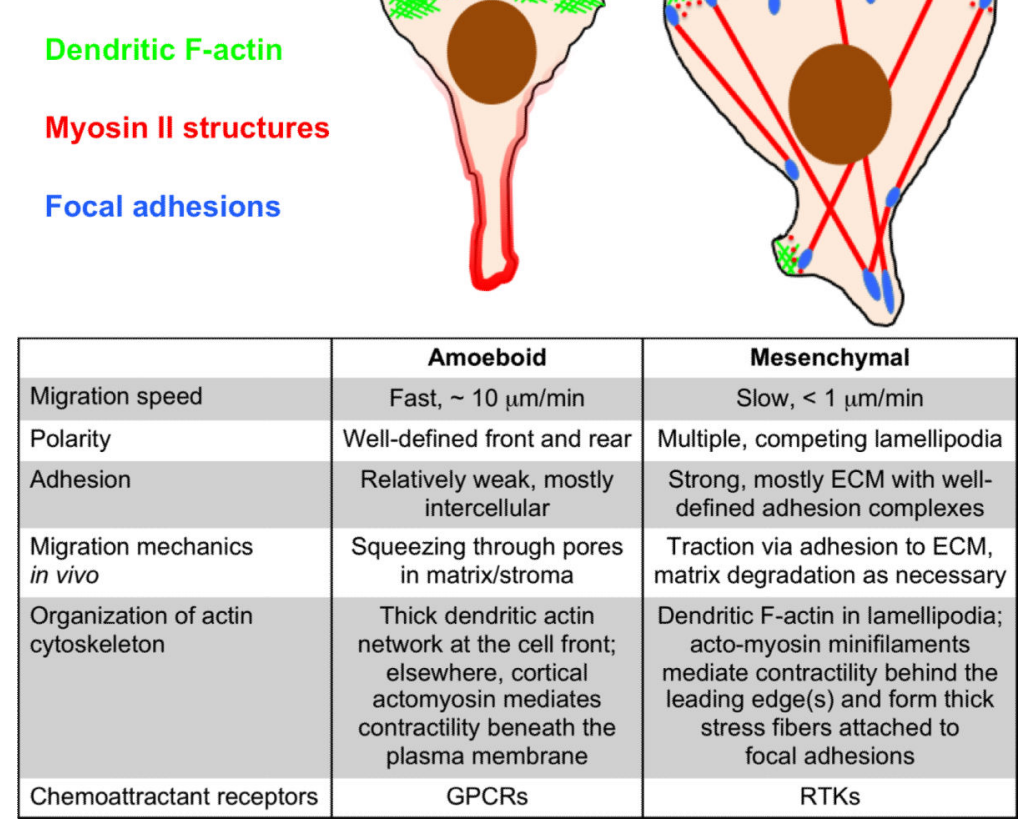

Figure 1. Mesenchymal vs. amoeboid motility and chemotaxis

The illustrations and table compare the structural and dynamic features of mesenchymal migration to those of amoeboid cells such as neutrophils and lymphocytes. 

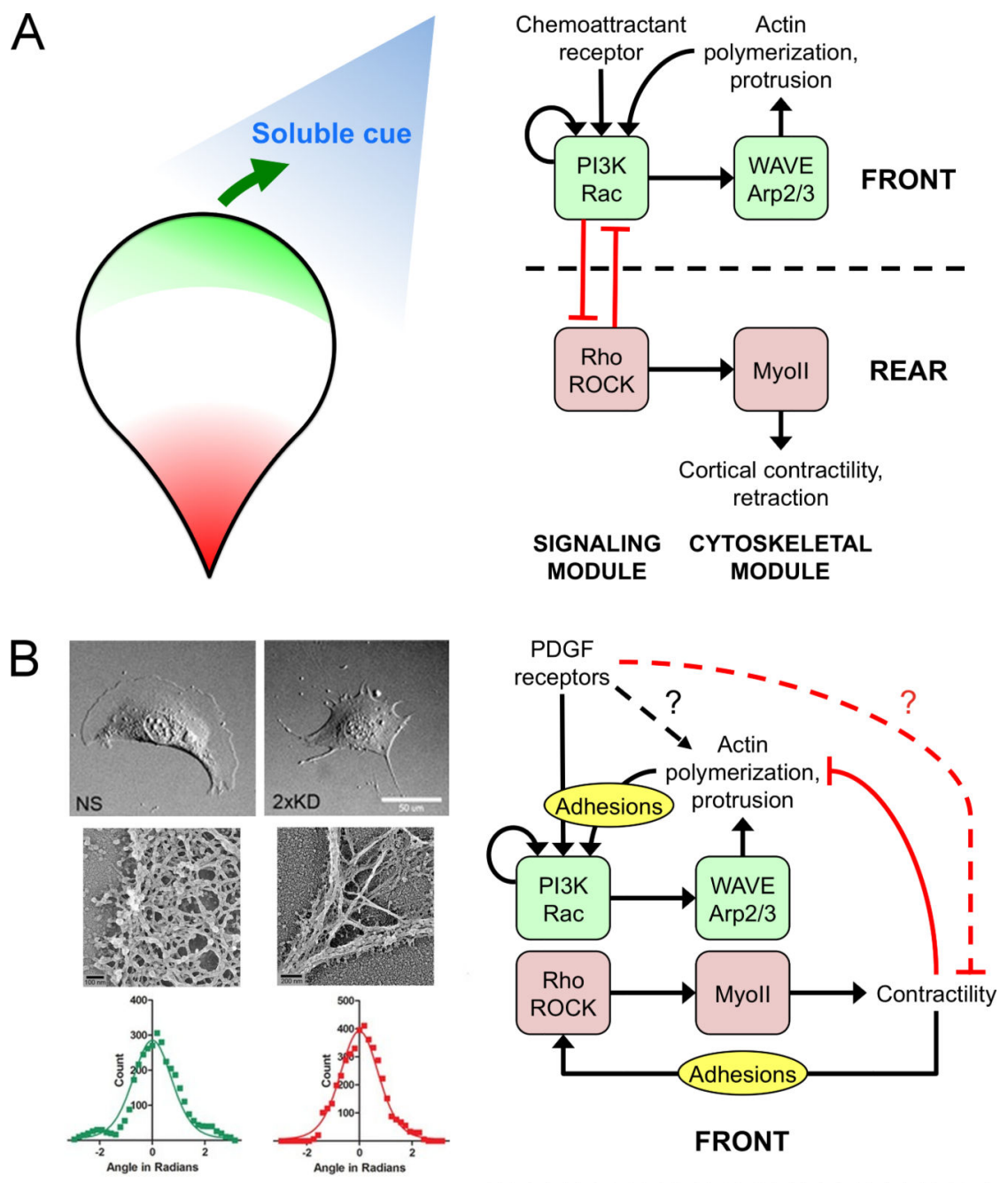

Figure 2. Clarifying the role of the PI3K/Rac/WAVE/Arp2/3 circuit in mesenchymal cells (A) In the conventional model of gradient sensing in amoeboid cells, PI3K and Rac are engaged in a signaling module that controls Arp2/3-mediated actin polymerization at the front of the cell. Cells establish and maintain polarity through positive feedback in this circuit, combined with its functional incompatibility with Rho signaling and active Myosin II (MyoII) at the cell rear. An external cue simply introduces a bias of the Arp2/3 circuit towards the left or right of the migration axis. (B) The alternative model of mesenchymal chemotaxis is spurred by the observation that depletion of Arp $2 / 3$ complex in fibroblasts results in loss of dendritic F-actin arrays associated with lamellipodia but does not affect chemotactic fidelity. Panels at left adapted from Wu et al. [55••] (Copyright 2012 Elsevier Inc., used with permission). In this model, the Arp $2 / 3$ circuit follows the cue of an as yet uncharacterized gradient sensing mechanism and is important for agile cell movement during chemotaxis as well as random migration. This functional distinction is consistent with the relative lack of polarization in mesenchymal cells, in which Arp $2 / 3$ and myosin modules are neighbors, and the role of adhesion complexes in activating them to elicit protrusion and retraction of lamellipodia, respectively. 


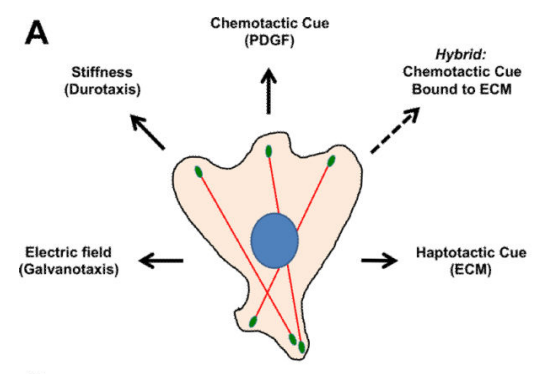

B
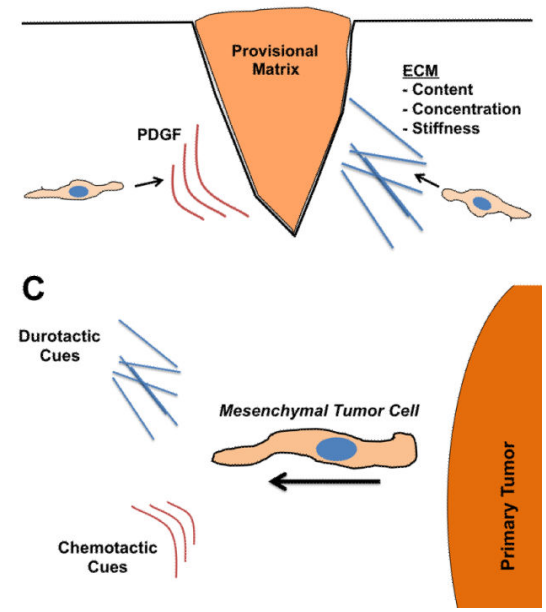

Figure 3. Directed migration cues for mesenchymal cells

(A) Diagram illustrating the diverse types of directional cues that mesenchymal cells respond to. Of note is the hybrid cue where chemotactic cues (e.g., growth factors) are bound to ECM scaffolds. (B) During cutaneous wound healing, fibroblasts (prototypical mesenchymal cells) respond to both PDGF (chemotaxis) and ECM cues (haptotaxis/ durotaxis). (C) Likewise, mesenchymal tumor cells emerging from primary tumors sense multiple directional cues. 